\title{
Beryllium abundance in turn-off stars of NGC $6752^{\star}$
}

\author{
L. Pasquini ${ }^{1}$, P. Bonifacio ${ }^{2,3,4}$, S. Randich ${ }^{5}$, D. Galli ${ }^{5}$, R. G. Gratton ${ }^{6}$, and B. Wolff ${ }^{1}$ \\ 1 European Southern Observatory, Garching bei München, Germany \\ e-mail: lpasquin@eso.org \\ 2 CIFIST Marie Curie Excellence Team \\ 3 Observatoire de Paris, GEPI, 92195 Meudon Cedex, France \\ ${ }^{4}$ INAF - Osservatorio Astronomico di Trieste, Trieste, Italy \\ INAF - Osservatorio Astrofisico di Arcetri, Firenze, Italy \\ 6 INAF - Osservatorio Astronomico di Padova, Padova, Italy
}

Received 17 August 2006 / Accepted 18 December 2006

\begin{abstract}
Aims. To measure the beryllium abundance in two TO stars of the Globular Cluster NGC 6752, one oxygen rich and sodium poor, the other presumably oxygen poor and sodium rich. Be abundances in these stars are used to put on firmer grounds the hypothesis of Be as cosmochronometer and to investigate the formation of Globular Clusters.

Methods. We present near UV spectra with resolution $R \sim 45000$ obtained with the UVES spectrograph on the $8.2 \mathrm{~m}$ VLT Kueyen telescope, analysed with spectrum synthesis based on plane parallel LTE model atmospheres.

Results. Be is detected in the $\mathrm{O}$ rich star with $\log (\mathrm{Be} / \mathrm{H})=-12.04 \pm 0.15$, while $\mathrm{Be}$ is not detected in the other star for which we obtain the upper limit $\log (\mathrm{Be} / \mathrm{H})<-12.2$. A large difference in nitrogen abundance $(1.6 \mathrm{dex})$ is found between the two stars.

Conclusions. The Be measurement is compatible with what found in field stars with the same $[\mathrm{Fe} / \mathrm{H}]$ and $[\mathrm{O} / \mathrm{H}]$. The "Be age" of the cluster is found to be $13.3 \mathrm{Gyr}$, in excellent agreement with the results from main sequence fitting and stellar evolution. The presence of Be confirms the results previously obtained for the cluster NGC 6397 and supports the hypothesis that Be can be used as a clock for the early formation of the Galaxy. Since only an upper limit is found for the star with low oxygen abundance, we cannot decide between competing scenarios of Globular Cluster formation, but we can exclude that "polluted" stars are substantially younger than "unpolluted" ones. We stress that the Be test might be the only measurement capable of distinguishing between these scenarios.
\end{abstract}

Key words. stars: abundances - stars: globular clusters: individual: NGC 6752 - stars: late-type - stars: individual: Beryllium

\section{Introduction}

In a recent paper we have successfully tested the use of beryllium (Be) as a cosmochronometer for the early phases of Galactic evolution (Pasquini et al. 2004). Be is produced by the interaction of Galactic cosmic rays (GCRs) with the interstellar medium, through the spallation of heavy element nuclei, most noticeably: carbon, nitrogen, and oxygen. Due to its origin, and in particular to the fact that GCRs are generated and transported globally on a Galactic scale, Be is expected to be characterized by a smaller dispersion than the products of type II supernovae, whose abundances in the early Galaxy are affected by the dispersed character of star formation and inefficient mixing of gas. This led to the suggestion that Be represents a "cosmic clock" (Suzuki et al. 2001; Beers et al. 2000). To test this hypothesis Pasquini et al. (2004) carried out the first measurements of Be abundances in a globular cluster (GC); namely they observed Be in two turnoff (TO) stars of the metal poor ([Fe/H] -2) GC NGC 6397, for which an independent age estimate was available. Be was detected in both stars at a level consistent with that of stars in the field with the same $[\mathrm{Fe} / \mathrm{H}]$ abundance. By comparing their Be values with models of Galactic evolution of $\mathrm{Be}$ as a function of time, they concluded that the cluster formed about 0.2 $0.3 \mathrm{Gyr}$ after the onset of star formation in the halo, in very good

* Based on observations collected at the ESO VLT, Paranal Observatory, Chile, program 075.D-0807(A). agreement with the cluster age derived from main sequence fitting. In order to prove that this result is not fortuitous, it is extremely important to carry out the same test on additional GCs.

As discussed by Pasquini et al. (2004), Be and Li measurements in GCs provide as well important constraints on the hotly debated issue of cluster formation. Detailed studies of chemical abundances in GC stars have revealed that anomalies are present in all the clusters studied, showing that they are not a homogeneous populations as far as the chemical composition of the stellar atmospheres is concerned (e.g. Gratton et al. 2004, and references therein). More specifically, whereas GCs appear to be extremely homogeneous in Fe and Fe-peak elements, star-tostar variations are seen for those elements involved in H-burning at high temperature. This behaviour is not present among field stars, which do not exhibit significant star-to-star variations in abundance ratios like $[\mathrm{O} / \mathrm{Na}]$ or $[\mathrm{Mg} / \mathrm{Al}]$ at a given overall metallicity. This intriguing abundance pattern, and in particular the fact that abundance anomalies are seen not only among evolved cluster stars but also in unevolved stars at the TO, suggests that part of the gas forming the stars that we observe today has been processed and ejected by a previous generation of stars. When considering that GCs are almost as old as the age of the Universe, this implies that this hypothetical previous generation might have been part of the elusive metal-free Population III, the first stars formed. By studying the current chemical composition of GC stars we may therefore try to determine the composition 
of the ejecta of the first stars and to understand their basic characteristics (mass, chemical composition).

In a series of papers we have investigated the light elements $\mathrm{Li}$ and $\mathrm{Be}$ in TO stars of GCs (Bonifacio et al. 2002; Pasquini et al. 2004, 2005a; Bonifacio et al. 2007, in preparation), showing that these elements are indeed very useful to investigate the process of GC formation. We recall that ${ }^{7} \mathrm{Li}$ and ${ }^{9} \mathrm{Be}$ are both destroyed at relatively low temperatures $(\sim 2.5$ and $\sim 3.5$ million $\mathrm{K}$ respectively) in stellar interiors. These temperatures are significantly below those at which typical reactions responsible for the chemical anomalies in GCs occur. Thus, in the hypothesis that a fraction of GC stars is born from the ejecta of a previous generation of stars, these stars should in principle show low Be and $\mathrm{Li}$ abundances. Note, however, that $\mathrm{Li}$ and $\mathrm{Be}$ form through different mechanisms. Whereas, as mentioned, ${ }^{9} \mathrm{Be}$ can only be produced by GCR spallation on the ISM, ${ }^{7} \mathrm{Li}$ is produced during Big Bang Nucleosynthesis, and the Galaxy is later enriched by several mechanisms, such as GCRs spallation, AGB stars, and, possibly, Novae (e.g., Travaglio et al. 2001).

Our observational results can be summarized as follows: the Li abundance is constant in NGC 6397 (Bonifacio et al. 2002), while Li is found to vary from star-to-star in NGC 6752 and 47 Tuc. In both these GCs, $\mathrm{Li}$ abundances are correlated with $\mathrm{O}$ and anti-correlated with $\mathrm{Na}$ abundances (Pasquini et al. 2005a; Bonifacio et al. 2006).

As for Be, the mere existence of Be in in the two TO stars of NGC 6397 suggests that the gas which formed the stars we now observe must have been sitting for at least a few hundred million years in the ISM exposed to the GCR spallation before the stars formed.

NGC 6397 could, on the other hand, be a somewhat "special" case, because it is one of the GCs where chemical anomalies are present at the lowest level, and, as mentioned above, the $\mathrm{Li}$ abundance is strikingly constant. It may therefore be questioned whether this cluster is a fair representative of all GCs. NGC 6752, on the opposite, is a prototype of GC with large chemical anomalies; these anomalies have been found all over the colour-magnitude diagram; the $\mathrm{Na}-\mathrm{O}$ anticorrelation has been discovered among main sequence stars (Gratton et al. 2001, hereinafter G01), as well as $\mathrm{Li}-\mathrm{Na}$ anticorrelation (Pasquini et al. 2005a). This cluster is therefore a very good prototype for anomalies; it has an intermediate metallicity $([\mathrm{Fe} / \mathrm{H}]=-1.48$, G01), and its age is virtually the same of NGC 6397 according to main sequence fitting (Gratton et al. 2003).

\section{Sample stars and observations}

Stars at the TO of NGC 6752 have been studied extensively for chemical abundances (Gratton et al. 2001, 2003; Carretta et al. 2005; James et al. 2004; Pasquini et al. 2005a). We selected two stars from the G01 sample having different composition; star 4428 is representative of the O-rich, $\mathrm{Li}$ rich component, while the star 200613 is representative of the Li poor component and, by inference, should be also oxygen poor. By observing stars at the extremes of the chemical distribution, we aimed at maximizing the chances of observing possible differences in Be abundance.

We recall that the Be lines are located at $313 \mathrm{~nm}$ and the Be observations of GC turnoff stars are very challenging, because the spectral region is crowded, the Be lines tiny and the UV flux of these relatively cool stars is low. When adding the relatively high absorption introduced by the earth's atmosphere, it clearly emerges that the TO stars of even the closest GCs are very faint for observations of Be lines. NGC 6752 TO stars are almost one magnitude fainter in the $V$ band than the NGC 6397 objects, but the relatively low reddening of the cluster $(E(B-V)=0.05$ : Gratton et al. 2003) allows a substantial flux recovery at the UV wavelengths, so that these observations, even if very demanding, are feasible with UVES at the VLT.

Table 1 summarizes the characteristics of the stars, including the abundances of the single elements, as derived from the literature. The stellar parameters listed in Table 1 are those adopted in our spectroscopic analysis. Only abundances of those elements which are known to vary from star to star $(\mathrm{O}, \mathrm{Na}, \mathrm{Li})$ are listed in Table 1; we also add in the table the results for $\mathrm{Be}$ and $\mathrm{N}$ as resulting from this paper. The reader can find additional element abundances in G01, James et al. (2004), and Carretta et al. (2005).

The observations were carried out in service mode at the VLT observatory in several runs during summer 2005 with the UVES spectrograph (Dekker et al. 2000). We used a 1" slit providing a resolving power of 45000 . The blue $\mathrm{CCD}$ was binned to minimize the CCD read out noise. In addition, all observations were taken at low airmass, to limit the atmospheric absorption, which is particularly high at these UV wavelengths. A total of 10 exposures of $90 \mathrm{~min}$ each were obtained per star. The observations were reduced using recipes from the UVES pipeline (Ballester et al. 2000). Given the fairly low S/N ratio of each observation in the Be region, we have been especially careful in reducing the data. The two major difficulties were identified in the correct estimate of the background and in the elimination of the "cosmic ray" events. We have therefore used two different reductions, either using the standard calibration and pipeline, or using the D lamp for flat fielding, which gives better spectra in the blue. This lamp, however, has some emission lines in its spectrum; it can be used in the spectral region of the Be lines, but not over the whole spectrum.

The reduced spectra were averaged leading to a $\mathrm{S} / \mathrm{N}$ ratio in the Be region of 20 and 10 per $0.025 \AA$ pixel for stars 4428 and 200613 respectively. The spectra in the Be region, together with our synthetic best fits (cf. next section) are shown in Fig. 1.

\section{Abundance analysis}

The method used has been described in Pasquini et al. (2004). A full synthesis of the region around the Be doublet has been performed using the line list given in Pasquini et al. (2004). For each star the best abundance was found by a $\chi^{2}$ fit to the whole feature. The best fits, shown in Fig. 1, correspond to Be abundances of $\log (\mathrm{Be} / \mathrm{H})=-12.04$ for the O-rich, Li-rich star 4428, while only an upper $\operatorname{limit}$ of $\log (\mathrm{Be} / \mathrm{H})<-12.2$ could be found for star 200613. Fitting errors were estimated with Monte Carlo simulations assuming a Poisson noise. The dispersion around the mean in 1000 Monte Carlo samples was of 0.04 dex for star 4428. These should be viewed as lower limit to the errors, since at these low $\mathrm{S} / \mathrm{N}$ ratios other sources of non-Poisson noise (e.g. shot-noise) could be important. To these errors associated to the noise in the data one must add the errors which derive from the uncertainty in the analysis procedure (atmospheric parameters, etc.). Since we are using lines of singly ionized Be, surface gravity is the parameter which most affects the Be abundance computation. The values of $\log g$ have been estimated by comparing the position of the stars in the color-magnitude diagram with theoretical isochrones. The uncertainty in such determination is dominated by possible errors in the distance moduli; we can then safely assume an error of $0.15 \mathrm{dex}$ in $\log g$ that translates into an uncertainty of $0.07 \mathrm{dex}$ in the $\mathrm{Be}$ abundance. The error arising from a change of $100 \mathrm{~K}$ in the effective temperature is about two 
Table 1. NGC 6752 sample stars, their atmospheric parameters and abundances. The atmospheric parameters, $[\mathrm{Fe} / \mathrm{H}],[\mathrm{O} / \mathrm{H}]$ and $[\mathrm{Na} / \mathrm{Fe}]$ are from $\mathrm{G01} ; \log (\mathrm{Li} / \mathrm{H})$ from Pasquini et al. (2005a), [N/Fe] and $\log (\mathrm{Be} / \mathrm{H})$ from this work.

\begin{tabular}{lllllllll}
\hline \hline Star & $T_{\text {eff }}$ & $\log g$ & {$[\mathrm{Fe} / \mathrm{H}]$} & {$[\mathrm{O} / \mathrm{H}]$} & {$[\mathrm{Na} / \mathrm{Fe}]$} & $\log (\mathrm{Li} / \mathrm{H})$ & {$[\mathrm{N} / \mathrm{Fe}]$} & $\log (\mathrm{Be} / \mathrm{H})$ \\
\hline & & & & & & & & \\
4428 & 6226 & 4.28 & -1.52 & -1.28 & -0.35 & 2.50 & 0 & $-12.04 \pm 0.15$ \\
200613 & 6226 & 4.28 & -1.56 & $/$ & 0.64 & 2.13 & 1.6 & $<-12.2$ \\
\hline
\end{tabular}

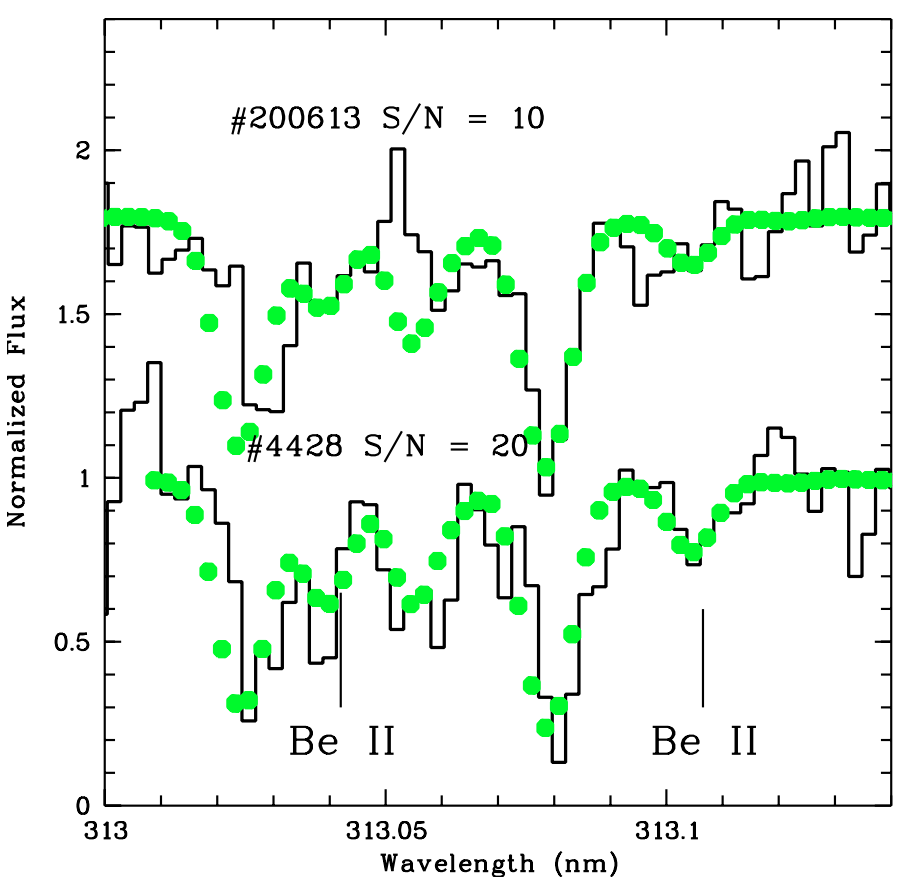

Fig. 1. UVES spectra of the two turn-off stars 200613 and 4428 of the GC NGC 6752. The dotted lines correspond to the Be abundances $\log (\mathrm{Be} / \mathrm{H})=-12.2$ (upper limit) for star 200613 and $\log (\mathrm{Be} / \mathrm{H})=$ -12.04 for star 4428 .

times lower: 0.03 dex. If we add these values to the errors due to noise ( 0.04 dex and 0.11 dex, respectively), we obtain an estimate of the total error on the Be abundance of 0.15 dex. Note that this error does not include possible systematic errors due to shortcomings and inadequacies of our modeling (model atmospheres, atomic data, etc.).

We recall that, while G01 used the same temperature for all NGC 6752 stars, given the strong dependence of Li on $T_{\text {eff }}$, Pasquini et al. (2005a) investigated the effects of adopting an additional temperature scale, and derived for these stars $T_{\text {eff }}$ from the $b-y$ color as well, finding $6013 \mathrm{~K}$ for star 4428 and $5948 \mathrm{~K}$ for star 200613. These temperatures, when entered into the ischrones of Straniero et al. (1997) imply slightly higher gravities for both stars, namely $\log g=4.43$ for star 4428 and 4.46 for star 200613. The combined effect of this change in atmospheric parameters is an increase of the Be abundance by 0.12 dex with respect to the values given in Table 1 .

We have also been able to measure the $\mathrm{N}$ abundance in these two stars, confirming that a very high $\mathrm{N}$ difference is present among them. This can be easily seen in Fig. 2, which shows the $\mathrm{NH}$ band at $336 \mathrm{~nm}$ for the two stars. Clearly their $\mathrm{N}$ abundance is different.

We estimate the nitrogen abundance by spectral synthesis of two NH UV bands, around $336 \mathrm{~nm}$ and around $340.5 \mathrm{~nm}$. Given the complexity of the bands it is not possible to carry out a formal fitting procedure of the whole spectral region. We have therefore inferred a "best abundance" by fitting the most

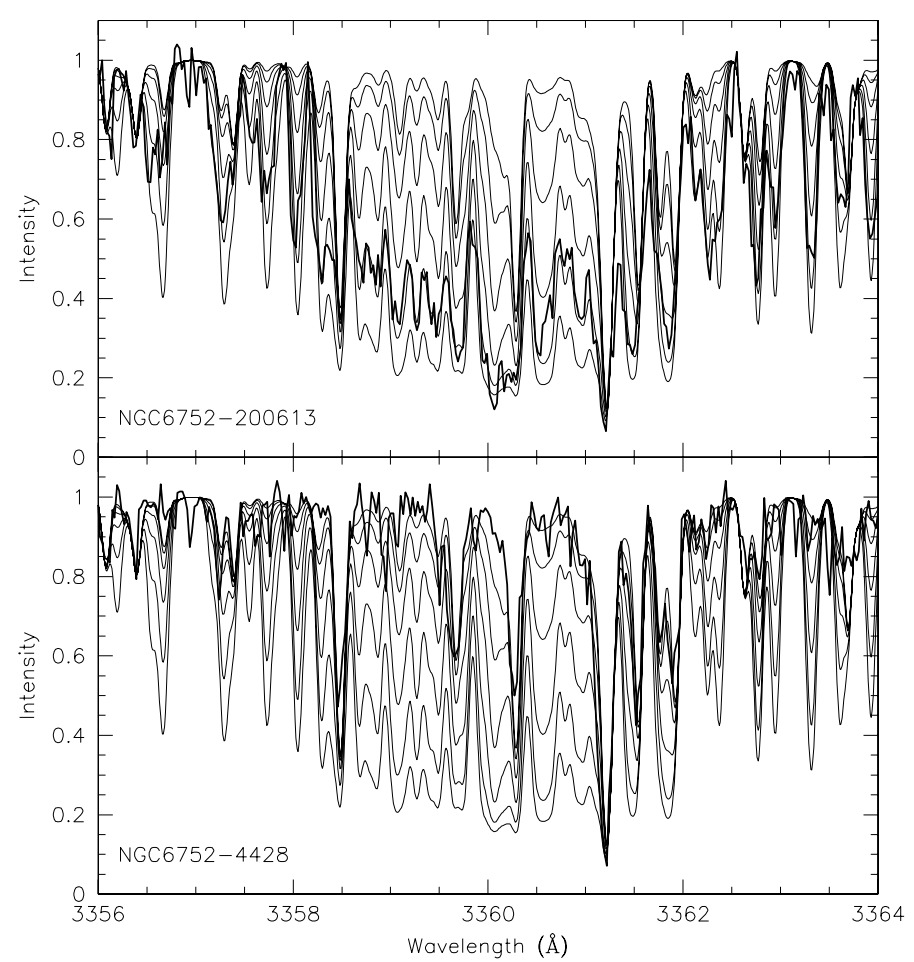

Fig. 2. UVES spectra of stars 200613 and 4428 around the NH band at $336 \mathrm{~nm}$. The band is much stronger in star 200613, indicating a strong $\mathrm{N}$ overabundance. Solid lines are the observed spectra; thin lines are synthetic spectra computed with parameters appropriate to the program stars, and $\mathrm{N}$ abundances of $[\mathrm{N} / \mathrm{Fe}]=0,0.4,0.8,1.2,1.6$ and 2.0.

prominent features. Despite several systematic and random uncertainties, we can compute a difference of about 1.6 dex in $\mathrm{N}$ abundance among the two stars. For star 200613 the value of $[\mathrm{N} / \mathrm{Fe}]=1.6$ is compatible with what found by Carretta et al. (2005) $([\mathrm{N} / \mathrm{Fe}]=1.7)$, who used different spectra and different spectral features (CN bands). As far as star 4428 is concerned, the value found $([\mathrm{N} / \mathrm{Fe}]=0)$ is 1 dex below that found by Carretta et al. (2005), which should be considered, however, as an upper limit rather than a detection $([\mathrm{N} / \mathrm{Fe}]=1.1)$.

The discrepancy between the two $[\mathrm{N} / \mathrm{Fe}]$ abundance of the "unpolluted" star 4428 is critical, since the evolution of nitrogen in GCs is one of the key data to understand the formation process of the clusters. In fact, while a value of $[\mathrm{N} / \mathrm{Fe}]=0$ is perfectly compatible with that found in metal-poor field stars, a value of $[\mathrm{N} / \mathrm{Fe}]=1$ (as determined by Carretta et al.) would be definitely much higher than that observed in un-evolved stars in the field, and it would point out to the need for a different source of $\mathrm{N}$ production in the formation process of GC. However, a high $\mathrm{N}$ abundance is clearly incompatible with the present spectra of this star.

Since the carbon isotopic ratio is a very sensitive diagnostic of mixing in stellar material which correlates well with $[\mathrm{N} / \mathrm{Fe}]$ ratios (Spite et al. 2006), it would therefore be interesting to cross-check the inference derived from $\mathrm{N}$ abundances with 
carbon isotopic ratios. Unfortunately we do not have ${ }^{12} \mathrm{C} /{ }^{13} \mathrm{C}$ ratios for these two stars, nor for any of the dwarf stars in this cluster since all the ${ }^{13} \mathrm{CH}$ features are too weak to be measured (Carretta et al. 2005).

An unusually high N value was found in one NGC 6397 unpolluted star (Pasquini et al. 2004) and a general $\mathrm{N}$ overabundance in $\mathrm{GC}$ has been postulated, for instance, by $\mathrm{Li}$ and Burstein (2003). [N/Fe] among field stars has been studied in the last years by several groups, which found that $[\mathrm{N} / \mathrm{Fe}]$ is approximately constant with metallicity in the range $-3.0 \leq[\mathrm{Fe} / \mathrm{H}] \leq$ 0.4 , as first shown by Carbon et al. (1987) and recently confirmed by Israelian et al. (2004) and Ecuvillon et al. (2004). According to our results, in NGC 6752, at odds with NGC 6397, the "unpolluted" star 4428 does not show evidence for strong $\mathrm{N}$ overabundance with respect to field stars; also for this element, abundances in field and "unpolluted" stars of NGC 6752 are extremely similar.

\section{Discussion}

\subsection{The age of NGC 6752 and comparison of Be in GC with field stars}

As mentioned in Sect. 1, the first detection of Be in a GC offered the opportunity to test the theoretical proposal that Be could be used as a cosmochronometer for the early evolution of the Galaxy. Adopting a standard model of GCRs spallation for the production of Be in the Galaxy, Pasquini et al. (2004) found an excellent agreement between the "Beryllium age" of NGC 6397 (13.4-13.5 Gyr, assuming a Galactic age of $13.7 \mathrm{Gyr}$ ), and the age of the cluster derived by Gratton et al. (2003) from main sequence fitting and theoretical isochrones $(13.5 \pm 1.1 \mathrm{Gyr})$. The use of $\mathrm{Be}$ as a cosmochronometer has been further exploited by Pasquini et al. (2005b) to study the halo-thick disk formation, finding some evidence for a faster star formation in the halo and a slower one in the disk. The detection of Be in the stars of a second GC, NGC 6752, characterized by a higher metallicity than NGC 6397, and presenting strong chemical inhomogeneities, is expected to provide additional constraints to the evolution of $\mathrm{Be}$ in the early Galaxy.

\subsection{The age of NGC 6752 and comparison of Be in GC with field stars}

It is clear that the use of $\mathrm{Be}$ as cosmochronometer requires a wider observational support. In this sense observations of additional GCs and new comparison between "Be" and "evolutionary" ages are necessary steps.

To this goal, we will adopt as the representative Be abundance of NGC 6752 the value of $\log (\mathrm{Be} / \mathrm{H})=-12.04 \pm$ 0.15 measured in star 4428 , which is the prototype of "unpolluted" stars in this cluster. With its high $\mathrm{Li}$ and $\mathrm{O}$, and low $\mathrm{Na}$, the atmosphere of this star is in fact expected to be composed mainly - if not entirely - of "unprocessed" gas (cf. next section).

In Fig. 3 we show the Be abundance vs. time according to the same Galactic chemical evolution model adopted in Pasquini et al. (2005b) (from Valle et al. 2002), together with the measured values of Be in NGC 6397 and NGC 6752. The assumed age for both clusters is the "isochrone age" derived by Gratton et al. (2003), 13.5 $\pm 1.1 \mathrm{Gyr}$ and 13.4 $1.1 \mathrm{Gyr}$ for NGC 6397 and NGC 6752, respectively. The age difference of 0.1 Gyr between the two clusters is much smaller than the error on the age determination. As in the case of NGC 6397, also for NGC 6752 the agreement with the model predictions is excellent,

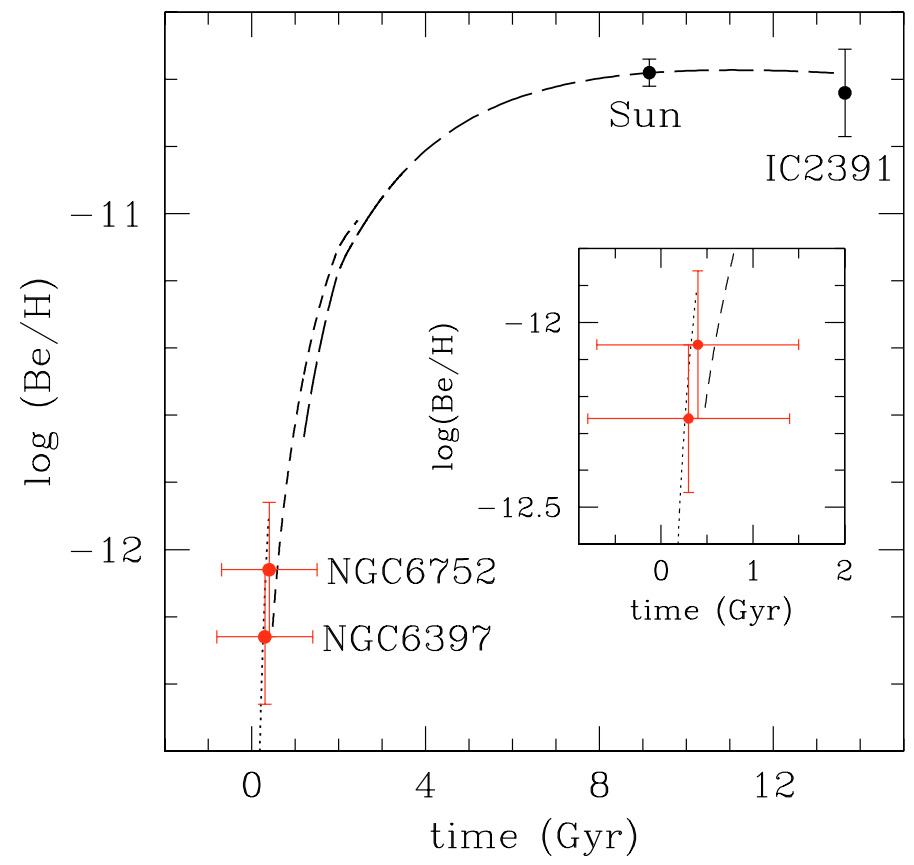

Fig. 3. Evolution of Be with time in the Galaxy according to a threezone Galactic chemical evolution model (Valle et al. 2002). The three curves refer to the halo, thick disk, and thin disk. The data points show the Be abundance in the young open cluster IC 2391 (age $55 \mathrm{Myr}$ ), the Sun (age 4.5 Gyr), the globular clusters NGC 6397 (isochrone age $13.5 \pm 1.1 \mathrm{Gyr}$ ) and NGC 6752 (isochrone age $13.4 \pm 1.1 \mathrm{Gyr}$ ). The model result is normalized to the solar meteoritic abundance. The inset shows a detail of the early Galactic evolution. The Be abundance of IC 2391 is from Randich et al. (2002); the Be abundances of the two GCs are from Pasquini et al. (2004) for NGC 6397 and from this work for NGC 6752. The isochrone ages are from Gratton et al. (2003).

reinforcing the validity of $\mathrm{Be}$ as an alternative (or additional) age estimator. A direct comparison between the observed and predicted abundance of Be in NGC 6752 would imply a "Be age" of 13.3-13.4 Gyr for this cluster, or about 0.1 Gyr less than the age of NGC 6397.

As we considered somewhat fortuitous the extremely good agreement between the "Be" and "isochrone" ages for NGC 6397, we also consider fortuitous the excellent agreement between the two age determinations for NGC 6752. It is worth noticing however, that, although the determination of the absolute value of the age of each cluster may be affected by large uncertainties with either method, the agreement in the relative age difference can be considered as a more robust result, as the effects of systematic errors should cancel.

Pasquini et al. (2004) noticed that the Be abundance of the NGC 6397 stars is fully consistent with the well-known Be vs. Fe trend (Gilmore et al. 1992; Molaro et al. 1997; Boesgaard et al. 1999, hereafter B99). In Fig. 4 we show the Be vs. Fe relation for the field stars from B99, together with the NGC 6397 points and the NGC 6752 value determined in this work. Figure 5 is similar, but $\mathrm{Be}$ is plotted as a function of $\mathrm{O}$ rather than Fe. Note that, as discussed by Pasquini et al. (2004), $\mathrm{O}$ abundances of field (B99) and cluster (G01) stars are on the same scale.

The agreement between the cluster and the field stars is impressive: they are indeed indistinguishable in the Be vs. Fe plane. In the Be vs. O plane the situation is slightly different, because the GC formation process depletes $\mathrm{O}$; this point will be discussed in the next session. However, insofar as "unpolluted" cluster 


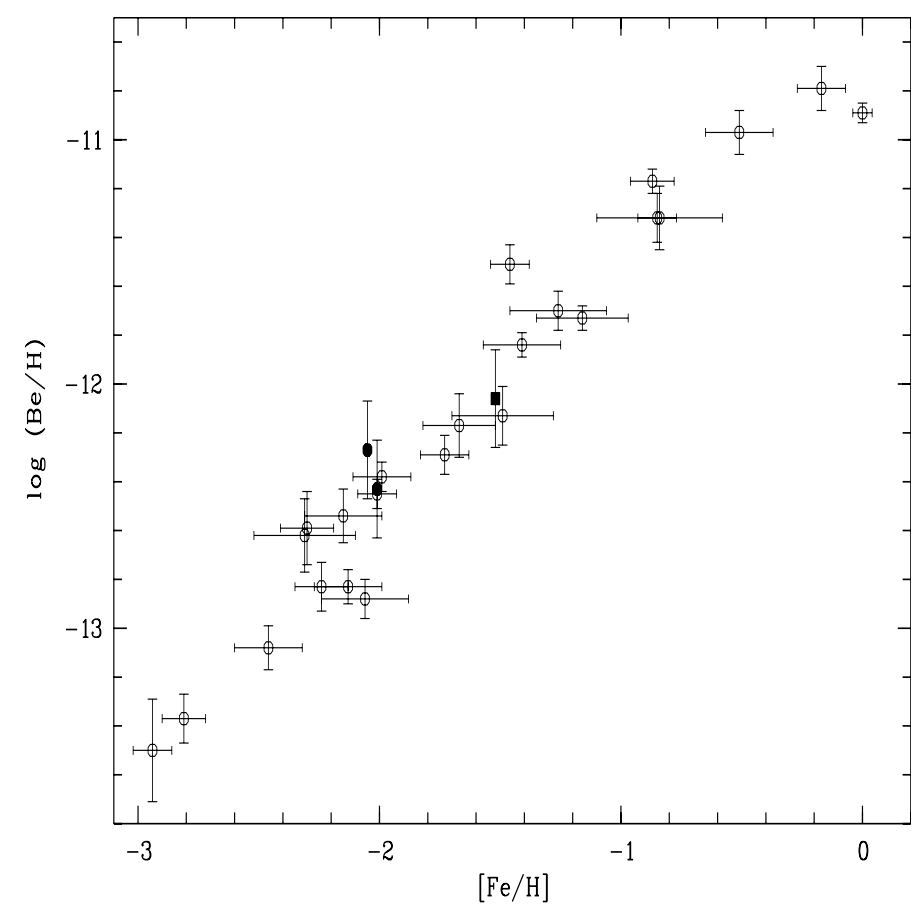

Fig. 4. Be abundance vs. Fe for the NGC 6397 (filled circles) and the NGC 6752 TO star (filled square) and the star from B99 (open circles). The stars in the clusters are indistinguishable from the field stars.

stars are considered, they are basically indistinguishable from field stars not only as far as nucleosynthesis elements are concerned, but this similarity extends to primordial nucleosynthesis products ( $\mathrm{Li}$ ) and to elements produced by GCRs spallation (Be).

A very simple conclusion is that Be production (or more precisely, the $\mathrm{Be} / \mathrm{Fe}$ ratio) seems to ignore the environment where the stars are born. We believe that this is a strong evidence in favour to the fact that the Be production in the early Galaxy is largely independent of the local ISM abundances; rather it reflects some global process, as we proposed in our previous work.

The approximately linear and tight relation between $\mathrm{Be}$ an Fe also suggests that their production sites are linked, and that they are also fairly independent of the formation site in the early Galaxy. The simplest explanation is that they are produced by the same type of events: supernovae, and that the medium was quite homogeneous at a given time, as far as $\mathrm{Fe}$ and $\mathrm{Be}$ are concerned.

It will be very difficult to prove the unicity of this solution, but the data collected so far are compatible with it. One test could be the observations of the Be lines for many stars of different populations and similar $[\mathrm{Fe} / \mathrm{H}]$; would the trends found by Pasquini et al. (2005a) be confirmed by larger samples of stars, not only the hypothesis of Be as a clock would be re-inforced, but also new insights about the mechanisms of halo and disk formation would be gained.

\subsection{Beryllium and globular cluster formation}

Our Be and $\mathrm{N}$ observations must be inserted in the general context of cluster chemical anomalies (see e.g. Gratton et al. 2004). In particular (see e.g. the discussion in Bonifacio et al. 2002; Pasquini et al. 2004; and Pasquini et al. 2005a), one of the possibilities of explaining the presence of Li in GC stars is that this element, destroyed in the previous generations of stars, has been also produced by them, for instance during the AGB phase,

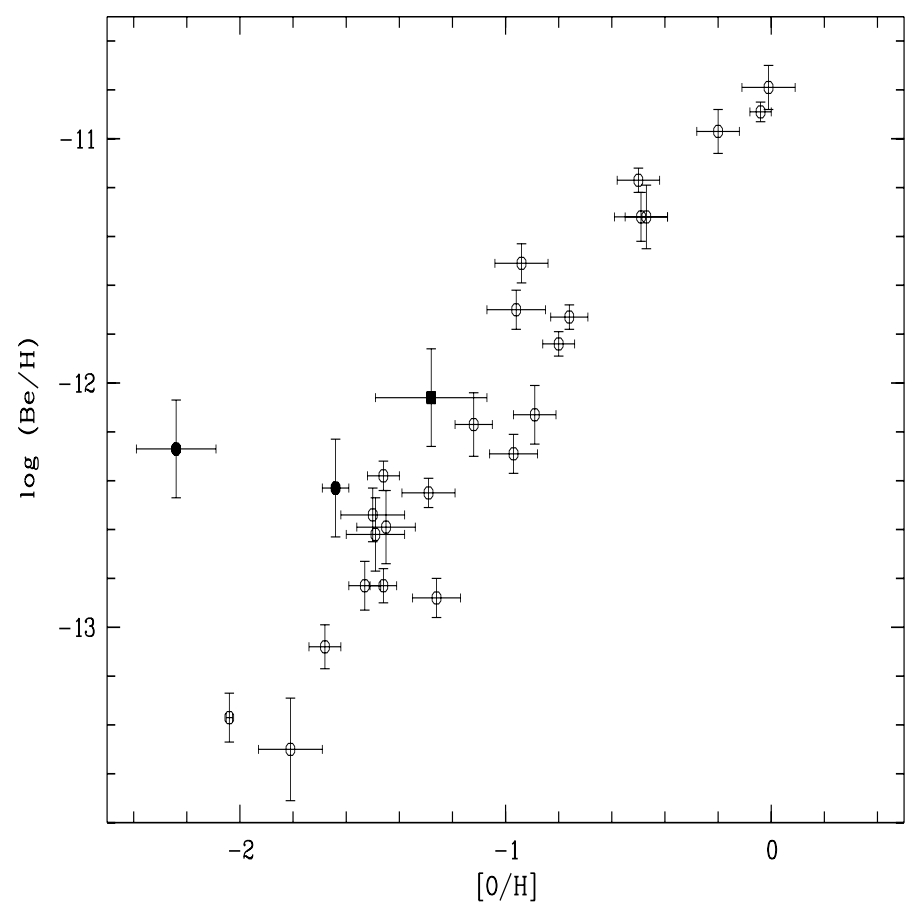

Fig. 5. Be abundance vs. O for the NGC 6397 (filled circles) and the NGC 6752 TO stars (filled square) and the star from B99 (open circles). The "unpolluted", O-rich stars in the clusters closely follow the field stars.

and brought to the surface by the so called Cameron- Fowler (Cameron \& Fowler 1971) mechanism.

Be cannot be produced in stars, so its mere detection in NGC 6752 (as well as in NGC 6397) shows beyond any doubt that the gas which formed the stars being here studied, has been sitting in a cloud and exposed to the GCRs for a few hundred million years.

In principle, at least for the NGC 6752 stars, one could invoke that the chemical composition we see now is the product of a more recent mixing (or mixture) of "pristine" (Be-rich) and "processed" (Be-poor) gas (see e.g. Charbonnel \& Prantzos 2006, for a similar approach).

The observations of NGC 6397 are difficult to be explained simply by a dilution scenario. The large $\mathrm{N}$ abundance observed in most stars of this cluster indicate transformations of a fraction of the original $\mathrm{O}$ into $\mathrm{N}$, in agreement with the only moderate excess of $\mathrm{O}$ (roughly a factor of about 2 smaller than in field stars having the same excess of $\alpha$-elements). This would suggest a similar dilution factor for the "pristine" material in that cluster, requiring a significant $\mathrm{Li}$ content in the "processed" material in order to recover the Spite plateau value, approximately observed in all NGC 6397 stars. Such a Li production would in turn favour the AGB stars as sources of the "processed" material. A further requirement is that this "processed material" should also be rich in Be, since the Be content of NGC 6397 is the correct one for its $[\mathrm{Fe} / \mathrm{H}]$ : this requires exposition to GCRs for a few $10^{8} \mathrm{yr}$. These coincidences seems rather hard to accept, mainly if present in several clusters.

On the other hand, the case of NGC 6752 seems easier. In this case, the most direct measure of the dilution of pristine material with processed ones in O-poor stars is provided by a comparison of the abundance of $\mathrm{O}$ with that observed in the most O-rich stars in the cluster, that we may assume to be composed of "unprocessed" material. Star 4428 is a good candidate for such group: indeed it has an abundance pattern similar to that of field 
stars of the same metallicity for all observed elements $(\mathrm{Be}, \mathrm{Li}$, $\mathrm{CNO}, \mathrm{Na}$, etc., if the $\mathrm{N}$ abundance provided by our robust $\mathrm{NH}$ observation is adopted). For what concerns stars made of "processed" material, unfortunately, the $\mathrm{O}$ abundance is not available for star 200613; however, we may assume for this star the same dilution factor we would obtain for stars having a similar composition, like e.g. star 4907 (G01, Carretta et al. 2005), for which $[\mathrm{O} / \mathrm{Fe}]=-0.25$. The difference in $\mathrm{O}$ content between stars 4428 and 4907 indicates a dilution factor of $\sim 4$. This is slightly more than the difference in the Li content $(\sim 3)$; however, this difference is likely within the observational uncertainties (while not excluding some fresh Li production). We should then expect a similar difference in the Be abundances. Our spectrum of star 200613 shows only an upper limit, that is fully compatible with a similar abundance of Be. Hence current observations of NGC 6752 may well be explained by a variable mixing of "pristine" and "processed" material, and the processed material could originate both in AGB stars and massive rotating ones. Again, the presence of $\mathrm{Be}$ indicates that the "pristine" material has been exposed to GCRs for a few $10^{8}$ yr. This strongly suggest that the material from which this cluster formed followed a chemical evolution similar to those of field stars of similar metallicity.

\subsection{Be abundance variations in the cluster?}

Due to measurement errors and low S/N of star 200613, our upper limit value does not allow us to exclude that its Be abundance is lower than that of star 4428, nor that, instead, the two stars have a similar Be content. We can however firmly exclude that it has a Be abundance which is a factor of 2 larger. The determination of a Be abundance difference between polluted and unpolluted stars is possibly the only way to understand how many generations of stars have preceeded the one we actually observe.

While it is well established that GCs have suffered by pollution from a previous generation of stars in the CNO elements and perhaps He (see e.g. Carretta et al. 2005; Piotto et al. 2005), it is not clear which progenitor has been responsible for the cluster chemical pollution. Several authors (e.g. Ventura et al. 2002; D'Antona et al. 2005) favour intermediate mass AGB stars, while others (Meynet \& Maeder 2006; Prantzos \& Charbonnel 2006) consider massive, rotating donors.

Both scenarios have in common that the polluters are a second generation of stars having the same metallicity of the cluster and composition of the field, which pollute the ISM in light elements but not in Fe. The "polluted" stars were a subsequent (third) generation, born from (or contaminated by) the ejecta of the second generation.

The unpolluted stars should therefore be "second generation" ${ }^{1}$ stars, similar in everything to the field stars, while the "polluted" objects are heavily contaminated, third generation. If this idea, common to both scenarios, were true, then we may expect a difference in Be abundance between the two generations of objects. The third generation of objects should have a younger age, and therefore a larger Be abundance. On the other hand the polluting material should be Be-depleted due to the high temperatures necessary to form the excess nitrogen. Therefore there are a priori three possibilities:

1. The third generation stars have higher Be abundance; in this case the age difference is large enough that a significant amount of Be has built up and/or the fraction of Be-depleted

${ }^{1}$ We adopt here the term second generation in a rather loose sense, simply to indicate that GC stars where preceded by several generations of stars. polluting material is small enough not to cancel this signature.

2. The third generation stars have lower Be abundance; in this case the age difference between the second and third generation is small and the pollution by Be-depleted material dominates.

3. The third generation stars have the same Be abundance as the second generation stars; this situation may arise in a number of ways by fine tuning the age difference between the two generations and the fraction of Be-depleted polluted material incorporated into the new stars; ranging from very small age difference and little pollution to large age difference and large pollution.

Our observations clearly rule out the first of the three cases, but the other two remain viable. The possibility of fine tuning age and pollution in the third case is however limited by the observations of $\mathrm{O}$ and $\mathrm{Li}$. From these two elements the dilution factor is in the range three to four and should be the same also for Be. Therefore, in principle, if the two stars had the same Be abundance we could estimate the age difference using this dilution factor and Fig. 3, assuming that the total amount of $\mathrm{CNO}$, necessary for the Be production, has not changed.

If the Be content of star 200613 were similar to that of the unpolluted star 4428 , for sake of completeness, we should mention that there is another scenario which could be envisaged: polluted and unpolluted stars belong to the same generation, and the metals and the $\mathrm{CNO}$ anomalies are produced by the same preceding generation, but perhaps by stars of different stellar masses. The previous generation of GC stars was extremely metal poor (perhaps even zero metal), dominated by stars of large masses; these exploded and ejected mass very quickly and their total mass was a substantial fraction of the GC total masses. The Supernovae contributed to the global enrichment of metals in the Galaxy, without leaving any local inhomogeneity, while CNO processing and ejection through the slower stellar winds, prior to $\mathrm{SNe}$ explosions, produced the differential enrichment in the cluster of $\mathrm{Na}, \mathrm{N}, \mathrm{Mg}$, and the depletion of $\mathrm{Li}$ in the cluster gas. It should be however noted, that this scenario requires that the SN explosions do not lead to a total mixing of the gas, which would cancel the inhomogeneities created by the winds. This may be difficult to reconcile with the cluster homogeneity in $\mathrm{Fe}$, which is better than $10 \%$. Additionally, it might be difficult to reconcile the required nucleosynthesis with current understanding of the yields from core collapse $\mathrm{SNe}$.

An accurate determination of the Be abundance in the polluted star 200613 should enable us to discriminate among competing scenarios.

Acknowledgements. We are grateful to the referee, R. Cayrel, for pointing out the consequences of excluding a large Be abundance in star 200613. P.B. acknowledges support from the MIUR/PRIN 2004025729_002 and from EU contract MEXT-CT-2004-014265 (CIFIST). We would like to emphasize as these observations are absolutely challenging and we thank the UVES team for building such a wonderful instrument and the VLT operations for carrying them on.

\section{References}

Ballester, P., Modigliani, A., et al. 2000, The Messenger, 101, 31

Beers, T. C., Suzuki, T. K., \& Yoshii, Y. 2000, in The Light Elements and Their Evolution, ed. L. da Silva, M. Spite, \& J. R. de Medeiros, PASP, IAU Symp., 198,425

Boesgaard, A. M., Deliyannis, C. P., King, J. R., et al. 1999, AJ, 117, 1549 (B99)

Bonifacio, P., Pasquini, L., Spite, F., et al. 2002, A\&A, 390, 91 (B02)

Bonifacio, P., Pasquini, L., et al. 2006, in preparation

Cameron, A. G. W., \& Fowler, W. A. 1971, ApJ, 164, 111 
Carbon, D. F., Barbuy, B., Kraft, R. P., Friel, E. D., \& Suntzeff, N. B. 1987, PASP, 99, 335

Carretta, E., Gratton, R. G., et al. 2005, A\&A, 433, 597

Charbonnel, C., \& Prantzos, N. 2006 [arXiv: astro-ph/0606220]

D'Antona, C., Bellazzini, M., et al. 2005, ApJ, 631, 868

Dekker, H., D' Odorico, S., Kaufer, A., Delabre, B., \& Kotzlowski, H. 2000, SPIE, 4008, 534

Ecuvillon, A., Israelian, G., Santos, N. C., et al. 2004, A\&A, 418, 703

Gilmore, G., Gustafsson, B., Edvardsson, B., \& Nissen, P. E. 1992, Nature, 357, 379

Gratton, R. G., Bonifacio, P., Bragaglia, A., et al. 2001, A\&A, 369, 87 (G01)

Gratton, R. G., Bragaglia, A., Carretta, E., et al. 2003, A\&A, 408, 529 (G03)

Gratton, R. G., Sneden, C., \& Carretta, E. 2004, ARA\&A, 42, 385

Israelian, G., Ecuvillon, A., et al. 2004, A\&A, 421, 649
Kurucz, R. L. 1993, CDROM, 13, 18

James, G., Francois, P., et al. 2004, A\&A, 414, 1071

Li, Y., \& Burstein, D. 2003, ApJ, 598, L103

Molaro, P., Bonifacio, P., Castelli, F., \& Pasquini, L. 1997, A\&A, 319, 593

Pasquini, L., Bonifacio, P., et al. 2004, A\&A, 426, 651

Pasquini, L., Bonifacio, P., et al. 2005a, A\&A, 441, 549

Pasquini, L., Galli, D., Gratton, R. G., et al. 2005b, A\&A, 436, L57

Piotto, G., Villanova, S., et al. 2005, ApJ, 621, 777

Randich, S., Primas, F., Pasquini, L., \& Pallavicini, R. 2002, A\&A, 387, 222

Spite, M., et al. 2006, A\&A, 455, 291

Suzuki, T. K., \& Yoshii, Y. 2001, ApJ, 549, 303

Travaglio, C., Randich, S., Galli, D., et al. 2001, ApJ, 559, 909

Valle, G., Ferrini, F., Galli, D., \& Shore, S. N. 2002, ApJ, 566, 252

Ventura, P., D’Antona, F., \& Mazzitelli, I. 2002, A\&A, 393, 215 